\title{
11 \\ Referencing and Citation for Graduate Students: Gain Without Pain
}

\author{
Ed S. Krol \& Lisa M. Krol \\ University of Saskatchewan
}

The objective of this paper is to share with other educators a teaching method that was developed to help graduate students, and potentially undergraduate students, understand how to properly reference and cite academic papers. In an attempt to teach rather than reprimand, a new teaching practice was developed for a graduate class at the University of Saskatchewan. The innovative assignment gave graduate students a short literature review to complete independently. The project then became a formative assessment of their referencing and citation skills. Student response was overwhelmingly positive. Upon successful completion, the instructor was assured of the students' understanding of appropriate referencing and citation practices.

\section{Introduction}

$\mathrm{I}_{\mathrm{o}}^{\mathrm{n}}$ nternational students are welcomed to institutions of higher learning for the wealth of new perspectives and diversity they bring to campus. However, these new perspectives can also bring cultural misunderstandings in academic practices. Referencing and citation is one such area that often causes confusion particularly where the practices of the new institution are vastly different from the student's prior academic experiences. Often violations by international students are not intended and simply result from a lack of familiarity with local expectations (Crocker \& Shaw, 2002; Pecorari, 2003). This is not to say that problems with referencing are limited to international students. With the vast amounts of information available electronically, many domestic students also have difficulty executing appropriate diligence in their written work (Parks, 2003). However, any such errors in referencing and citation can cause serious consequences for students which may include grade penalties, failure, or expulsion.

As an instructor of a graduate course in pharmaceutical sciences, I have struggled to communicate the importance and mechanics of proper citation and referencing to all graduate students but especially to international students. In an effort to reduce plagiarism issues, I offered to pre-read assignments prior to their due date and give students feedback on their structure, writing 
style, and analysis. When reviewing these drafts, I frequently came across plagiarism and incorrect or inconsistent referencing techniques. In drawing these errors to the students' attention, it was clear that in many cases the students were unaware of their violations or the very serious potential consequences of their infractions. Oftentimes, even with first draft feedback the final products continued to exhibit significant errors and the students themselves seemed confused and frustrated. It seemed unfair to pursue academic penalties when often the cause of error was and continued to be misunderstanding.

Faced with this dilemma, I found several possible solutions: continue to provide feedback on first drafts of assignments, give heavier academic penalties for infractions, or develop a training method to demonstrate the importance and mechanics of proper citation. The following describes a new instructional model developed and implemented to help improve student academic writing skills.

\section{The Writing Sample Assignment}

I decided that students would submit a writing sample to demonstrate their abilities; however, the assignment would need to possess several unique features. First, the assessment of the sample could not be punitive while the students were learning appropriate techniques. However, there still needed to be consequences for not successfully completing the task to ensure that all students met a minimum standard. The task itself could not be onerous as it was not the main focus of the course. The task also had to apply to a wide range of science backgrounds as students from various departments enrol in this particular class. And finally, it was also important to design a project that would be authentic to students' academic and science training.

The Writing Sample Assignment grew from these characteristics. I first ask the students to write a two-page review article on a given topic. The topics I choose are purposefully quite general. I then provide3-4 short academic articles for students to read which must be referenced in the final assignment. I choose articles for their general accessibility again because students come from a variety of different disciplines. Students submit their work electronically. Electronic submission facilitates assessment of issues of plagiarism as I can easily search the Internet for suspect phrases. Extensive feedback is provided on the first draft, particularly in the areas of referencing and citation. Students correct the work and resubmit the assignment for additional feedback until I am satisfied that the student can competently reference and cite their own work.

This approach meets my first intention in that it is not punitive because students are given unlimited opportunities to improve their work without academic penalty for plagiarism or other mechanical issues. Although this assignment does not contribute to the students' final grade in the course, they must achieve a PASS to guarantee adherence to a minimal standard. To ensure participation and completion of the assignment, the course syllabus states that the Writing Sample Assignment must be satisfactorily accomplished prior to submitting any other work for credit. My intention of this last requirement is to encourage students to use the knowledge gained in their first assignment for the betterment of subsequent course work.

The assignment is limited to two single-sided pages or approximately 1000 words. The paper could conceivably be written in six hours or less, and is not meant to be onerous or detract from the content of the course. Examples of topics that I have chosen include: the link between autism and childhood vaccinations, and the effects of components of red wine on cardiovascular health. Most of the articles given to the students for background are brief -four pages or less - and students can include additional references but are not required to do so.

One factor I stress to students is that any details found in the articles given have to be verified with the primary source before being included in the students' own writing. Although not originally intended as an outcome of the assignment, students come to better understand the research and the literature review process.

Finally, I provide specific format guidelines which include font, margins, pagination, title, subtitles, and examples of reference style and 
format. These instructions are given to replicate the conditions of thesis and manuscript submissions. Errors involving any of the above are sufficient to require resubmission. While such errors are often seen by students as trivial, it is important to emphasize the necessity of attention to detail to ensure that manuscripts and theses meet all technical requirements to avoid rejection.

Some students are quite successful with the assignment and receive a PASS on the first attempt. Other students struggle and require extensive remediation and may have to resubmit numerous times. Where possible I assist students and recommend various campus resources where they might obtain further assistance for specific writing problems. This assignment has now been adopted in all of my graduate classes and every student in each class must complete the assignment regardless of whether or not they have taken another course with me and have already completed a Writing Sample. I have found that students repeating the assignment frequently need the reminder, although they are generally more proficient than those doing the work for the first time.

Recently, this methodology was included in a new offering from our College of Graduate Studies and Research: Canadian Academic Acculturation and Literacy for International Graduate Students was developed to help acclimatize newly arrived international students to research expectations and culture of the Canadian university environment. Participating students recognized that they were largely unaware of the expectations that they would be held to prior to the Writing Sample Assignment. For example, some students expressed surprise at having to cite so much material while others indicated that they were taught in their home cultures to use words verbatim from the literature as a way of demonstrating respect. Students were most appreciative of the opportunity to learn in this nonjudgemental and hands-on manner.

Additionally, I have had discussions with instructors from McMaster University and determined that they are implementing a similar strategy. In their introductory undergraduate course, Inquiry for Chemical Biology, 10\% of the students' course grades are devoted to various exercises which include elements of literature research techniques and writing mechanics (McKenzie \& Berti, 2011). Many faculty in Chemical Biology at McMaster University have concluded that the communication of expectations and explicit instruction is necessary for undergraduate students to achieve competency in scientific writing. In essence, they assume students have no previous knowledge of scientific writing and they seek to demonstrate appropriate techniques. In contrast, the Writing Sample Assignment determines graduate students' base competencies with scientific writing and then creates opportunity for instruction and, in extreme cases, remediation. Clearly, many undergraduate and graduate students do not have a sufficient grasp of academic writing but by explicitly teaching and reinforcing these skills early, students have the opportunity to practice throughout their course of study. The final outcome of these efforts will presumably be manifested in improved quality of theses, manuscripts, senior undergraduate research reports, and projects.

\section{Challenges}

Ongoing challenges with this Writing Sample method include students compartmentalizing the knowledge they have acquired during the assignment to one particular instance. In some cases, students who have completed the Writing Sample Assignment successfully repeat the same errors in subsequent assignments in the same course or carry errors over to theses, manuscripts, and research reports outside of the class. Currently, the College of Pharmacy and Nutrition is considering making the Writing Sample Module a mandatory component for all incoming graduate students. It is hoped that such an extension of the project will help students to generalize the concepts to all areas of their work. The other significant challenge to this approach is the time involved for both the instructor and the student. This method will only be successful where the instructor can dedicate time both to the reading of the assignments and also to the explicit instruction of writing mechanics where necessary. Currently the 
instructor to student ratio is 1 to 5 and each paper takes no more than 20 minutes to grade and is quite manageable. For students, the time investment for a project that does not contribute to their grade is somewhat difficult to understand initially. However, once they complete the process and refine the assignment they do begin to appreciate how their invested time is recouped with the knowledge gained and they realize that they become more efficient in preparing larger documents.

\section{References}

Crocker, J. \& Shaw, P. (2002). Research student and supervisor evaluation of intertextuality practices. Hermes Journal of Linguistics, 28, 39-58.

McKenzie, N. \&Berti, P. (2011, June). The ongoing evolution of a second yearundergraduate chemical biology inquiry course: A guided inquiry approach to facilitate self-directed learning.Presented at STLHE Conference, Saskatoon, Saskatchewan, Canada.

Parks, C. (2003). In other (people's) words: Plagiarism by universitystudents-literature and lessons. Assessment and Evaluation in HigherEducation, 28, 471-488.

Pecorari, D. (2003). Good and original: Plagiarism and patchwriting in academic secondlanguage writing. Journal of Second Language Writing, 12, 317-345.

\section{Biographies}

Ed S. Krol is an Associate Professor in the College of Pharmacy and Nutrition at the University of Saskatchewan. He has become increasingly involved in the development of methods to help improve graduate student writing skills, particularly for international students.
Lisa M. Krol is the Curriculum Coordinator for the University of Saskatchewan Language Centre. She regularly consults on the needs of international students as related to various projects across campus. 